CLINICAL STUDY

\title{
Increased risk of thyroid pathology in patients with thyroid hemiagenesis: results of a large cohort case-control study
}

\author{
Marek Ruchala, Ewelina Szczepanek, Witold Szaflarski ${ }^{1}$, Jerzy Moczko ${ }^{2}$, Agata Czarnywojtek, Leszek Pietz, \\ Michal Nowicki ${ }^{1}$, Marek Niedziela ${ }^{3}$, Maciej Zabel ${ }^{1}$, Josef Köhrle ${ }^{4}$ and Jerzy Sowinski \\ Department of Endocrinology, Metabolism and Internal Medicine, University of Medical Sciences, Przybyszewskiego Street 49, 60-355 Poznań, Poland, \\ ${ }^{1}$ Department of Histology and Embryology, University of Medical Sciences, H. Święcickiego Street 6, 61-781 Poznań, Poland, ${ }^{2}$ Department of Computer \\ Science and Statistics, University of Medical Sciences, Dębrowskiego Street 79, 60-529 Poznań, Poland, ${ }^{3}$ Department of Pediatric Endocrinology and \\ Diabetes, University of Medical Sciences, Szpitalna Street 27/33, 60-572 Poznań, Poland and ${ }^{4}$ Institut für Experimentelle Endokrinologie, Charité \\ Universitätsmedizin Berlin, Augustenburger Platz 1, D-133535 Berlin, Germany
}

(Correspondence should be addressed to M Ruchala; Email: mruchala@ump.edu.pl)

(M Ruchala and E Szczepanek contributed equally to this work)

\begin{abstract}
Objective: Thyroid hemiagenesis (THA) is an anomaly resulting from the developmental failure of one thyroid lobe. Etiopathogenesis, clinical significance, and management of patients in whom THA is diagnosed are still a matter of debate. The aim of the study is to provide the first systematic analysis of a large cohort of subjects with THA.

Design: Forty patients with THA are described in comparison to a control group of 80 subjects with fully developed thyroid gland.

Methods: Serum concentrations of thyrotropin (TSH), free thyroxine $\left(\mathrm{FT}_{4}\right)$, free triiodothyronine $\left(\mathrm{FT}_{3}\right)$, and thyroid autoantibodies were measured. In 37 patients, thyroid ultrasonography and Tc-99m thyroid scintiscan were performed, followed by fine-needle aspiration biopsy if indicated. The remaining archival three cases were diagnosed with the use of I-131 scintiscan under basal conditions and after TSH stimulation.

Results: Patients with THA, while usually clinically euthyroid, presented with significantly higher levels of $\mathrm{TSH}$ and $\mathrm{FT}_{3}$ as well as with higher $\mathrm{FT}_{3} / \mathrm{FT}_{4}$ concentration in comparison to the control group. Furthermore, a higher incidence of associated functional, morphological, and autoimmune thyroid disorders in patients with THA was observed when compared to subjects with bilobate thyroid $(P<0.05)$.

Conclusions: Our results revealed that individuals with THA are more likely to develop thyroid pathology. The observed high incidence of associated pathologies is presumably due to long-lasting TSH overstimulation. Therefore, THA diagnosis should be followed by systematic observation and adequate levothyroxine treatment in patients with elevated TSH level.
\end{abstract}

European Journal of Endocrinology 162 153-160

\section{Introduction}

Thyroid hemiagenesis (THA) is a rare inborn anomaly, which occurs if one of the thyroid lobes fails to develop. Because of a mostly asymptomatic course, THA is usually detected incidentally while investigating concomitant thyroid pathologies or during screening examinations (1-3). Due to the development and increasing accessibility of various imaging techniques, more cases are detected. However, the etiopathogenesis of this condition, its clinical significance, impact on thyroid function, and development of associated thyroid pathologies as well as the management of patients in whom the anomaly is diagnosed are still a matter of debate $(1,4-7)$. To date, individual case studies have been reported most often, mainly as an anatomical curiosity or as a diagnostic dilemma, whereas the literature lacks methodological studies of large groups of patients presenting THA, which would provide objective answers to these controversies $(4,5,8-13)$. Since hormone production by a single thyroid lobe is sufficient to maintain clinical euthyroidism, provided no concomitant thyroid pathology exists, THA has been up to now regarded as a rather benign developmental variant requiring no treatment.

Our study provides the first systematic analysis of a large cohort of subjects diagnosed with THA. The group of 40 patients was analyzed according to a clinical, hormonal, morphological, and immunological profile in comparison to a control group of subjects with normally developed thyroid gland. 


\section{Subjects and methods}

\section{Subjects}

The studied group consisted of 40 patients newly diagnosed with THA, aged between 12 and 79. Except the first three archival patients, the study was designed prospectively and all subjects diagnosed with THA at the thyroid ultrasound unit in our department between January 2002 and December 2008 were involved in the study. The patients were included into the studied group after the diagnosis of congenital absence of one lobe on thyroid ultrasound. Each time, THA diagnosis was confirmed by thyroid scintiscan. The patients were referred for thyroid ultrasound due to various reasons. In 17 patients, the anomaly was detected accidentally during screening examination or evaluation of nonthyroidal disorders. The remaining subjects at admission presented one or more of the following thyroid-related symptoms: asymmetry of the thyroid gland, detected by the patient himself/herself or during physical examination (22 patients) and symptoms of mild thyroid dysfunction - hypothyroidism (eight patients) or hyperthyroidism (six patients).

The control group of 80 persons with bilobate thyroid gland, matched for age and gender, was selected randomly from 2159 participants of a cross-sectional population-based thyroid screening program, which was conducted simultaneously at our department.

All examined subjects live in the same region of Poland (Wielkopolska), which is classified by the WHO as a mild iodine-deficient area with median iodine urinary excretion at the level of 50-99 $\mu \mathrm{g} / \mathrm{l}(14)$.

\section{Serum assays}

The hormonal assessment included the measurement of serum TSH, free triiodothyronine $\left(\mathrm{FT}_{3}\right)$, and free thyroxine $\left(\mathrm{FT}_{4}\right)$, and was performed using Hitachi Cobas e601 chemiluminescent analyzer (Roche Diagnostics). Patients were classified as euthyroid when TSH level was within normal ranges $(0.27-4.2 \mu \mathrm{IU} / \mathrm{ml})$. Hypothyroidism was defined as TSH level above the reference values, while hyperthyroidism was diagnosed when TSH concentration was below the normal level. Thyroid autoantibodies concentrations (thyrotropin receptor antibody, TRAb; antithyroid peroxidase antibody, TPOAb; and antithyroglobulin antibody, $\mathrm{TgAb}$ ) were assessed by radioimmunological method with the use of commercially available BRAMHS anti-TPO, anti-Tg, and TRAK RIA kits, and scintillation gamma counter (LKB Wallac CliniGamma 1272).

\section{Thyroid imaging}

Thirty-seven prospectively analyzed patients with THA and 80 control subjects underwent thyroid ultrasonography (US) performed by the same experienced sonographist with a $17 \mathrm{MHz}$ linear probe using the ALOKA SSD 3500 SV instrument. The hemiagenetic thyroid volume was additionally measured by the mean of elliptical shape volume formula $(\pi / 6 \times$ length $\times$ width $\times$ depth). Subsequently, it was compared to the regular size of one thyroid lobe, which was considered as half of the normal total thyroid volume $(<18 \mathrm{ml}$ in women and $<25 \mathrm{ml}$ in men, WHO recommendations). Thyroid volume of patients with THA was compared at first with the total thyroid volume of the control subjects, and then with the average volume of one thyroid lobe, considered half of the total thyroid volume of subjects with bilobate thyroid. Moreover, any abnormality detected on US was also reported. Additionally, all patients with THA underwent Tc-99m thyroid scintiscan. In the remaining three retrospectively analyzed patients, the diagnosis of THA was made entirely with the use of I-131 scintiscan under basal conditions and after TSH (Ambinon, Organon, Oss, Holland) stimulation.

\section{Cytological examination}

Thyroid imaging was followed by fine-needle aspiration biopsy (FNAB) of detected lesions, if indicated. The examination of obtained specimens was performed in the Department of Pathology, Poznan University of Medical Sciences, by two pathologists as a routine medical procedure.

\section{Statistical analysis}

First, the group of patients with THA diagnosed before the age of 25 was compared with the subjects in whom the anomaly was diagnosed later. Secondly, particular features and incidence of specific abnormalities in patients with THA were compared to those in a control group of subjects with fully developed thyroid gland. The significance of differences of the parameters measured in an interval scale was performed either with Student's t-test for unpaired data or with Welch test. Additionally, obtained results were confirmed using distribution-free Mann-Whitney test. Categorical data measured in nominal scale presented in $2 \times 2$ contingency tables were analyzed using Fisher's exact test. Linear relationship between analyzed variables measured in an interval scale was estimated using Pearson's correlation coefficient. All calculations were done with STATISTICA version 8.0. Assumed significance level was equal to 0.05 .

The above-mentioned procedures are in accordance with the Helsinki Declaration of 1975 as revised in 2000. The study was approved by the local ethical committee, and all patients gave informed consent to participate. 

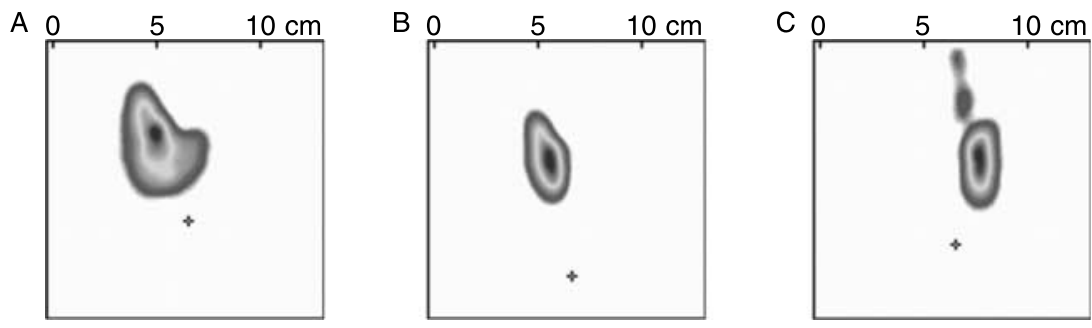

Figure 1 Three different types of thyroid hemiagenesis visualized by Tc-99m thyroid scintigraphy. The marker $(+)$ indicates the suprasternal notch. (A) Isolated left lobe agenesis (hockey stick sign), (B) associated left lobe and isthmus agenesis, and (C) associated right lobe and isthmus agenesis with pyramidal lobe.

\section{Results}

In the studied group, a considerable prevalence of women with THA was noted, with 7:1 female to male ratio. Out of the 40 patients, 35 presented left-sided agenesis. In two patients, a marked pyramidal lobe was visualized. Isthmus was absent in all the five patients with right-sided agenesis and in seven patients with left lobe agenesis. Isolated agenesis of left lobe (28 out of 40 patients) was a predominantly observed type of THA. The remaining patients presented left lobe and isthmus agenesis, or right lobe and isthmus absence (Fig. 1).

THA was very often associated with several thyroid pathologies (Fig. 2). The most frequent associated thyroid disorders were thyroid nodules and autoimmune thyroid diseases. Simple goiter and nonautoimmune subclinical hypothyroidism were less often observed. Patients were usually euthyroid (26 persons); however, hypothyroidism was observed in ten subjects, and hyperthyroidism in the remaining four subjects (Table 1).

The thyroid lobe size was within normal ranges in only 9 out of 40 patients. In the remaining 31 patients, it was enlarged compared to half of the normal total thyroid volume, while 12 of them fulfilled goiter criteria for bilobate thyroid gland.

The FNAB of detected lesions was performed in 22 patients. On FNAB, benign lesions (normal thyrocytes and cystic or colloid nodules) were diagnosed in 16

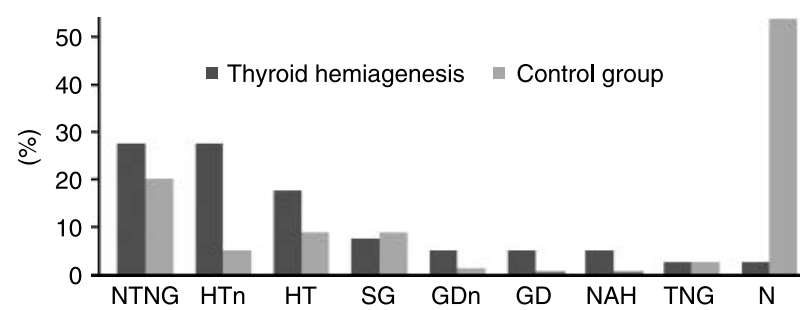

Figure 2 The incidence of associated thyroid pathologies in patients with thyroid hemiagenesis and control group. GD, Graves' disease; GDn, Graves' disease, nodular variant; HT, Hashimoto's thyroiditis; HTn, Hashimoto's thyroiditis, nodular variant; N, no associated thyroid pathology; $\mathrm{NAH}$, nonautoimmune hypothyroidism; NTNG, nontoxic nodular goiter; SG, simple goiter; TNG, toxic nodular goiter. patients. In four patients, the FNAB was nondiagnostic. Oxyphilic nodules were detected in the remaining two patients. Although there were no malignant lesions found on cytological examination, surgical treatment was recommended to six patients due to the following reasons: a 'suspicious' sonographic appearance, nondiagnostic FNAB findings, toxic nodular goiter, and a high goiter volume.

Patients with THA were subsequently divided into two groups - with early ( $\leq 25$ years old) and late $(>25$ years old) diagnoses of the anomaly. The comparison of these two groups revealed significant differences in thyroid volume, presence of heterogeneous decreased echogenicity on US, and incidence of increased TPOAb. No significant differences in gender; $\mathrm{TSH}_{1} \mathrm{FT}_{4}$, and $\mathrm{FT}_{3}$ concentrations; or incidence of focal lesions were found (Table 2). Out of the 40 subjects with THA with absent thyroid autoantibodies, 18 were selected for hormonal analysis in order to exclude the influence of autoimmune disturbances on their hormonal profile. In the same group, a significant positive correlation between thyroid volume and the age of diagnosis $(P=0.009)$ was also noted. However, a negative correlation between thyroid volume and age did not reach statistical significance $(P=0.218)$.

The comparison of THA group to control subjects with bilobate thyroid, matched for age and gender, revealed significantly increased $\mathrm{TSH}$ and $\mathrm{FT}_{3}$ concentrations (Table 1). However, no significant difference in $\mathrm{FT}_{4}$ concentration was detected. $\mathrm{FT}_{3} / \mathrm{FT}_{4}$ ratio was found to be higher in patients with THA than in controls (Table 1). Similarly, a comparison of hormonal profile was performed for 18 THA patients with no autoimmune disorder.

Patients with THA were significantly more often found to be hypothyroid, while incidence of hyperthyroidism was similar in both the groups (Table 1). The comparison between patients with THA and the control group demonstrated no significant difference in the total thyroid volume. Thus, the total hemiagenetic thyroid volume was significantly higher in the THA group when compared to the mean volume of one thyroid lobe (half of the total thyroid volume in control subjects). Higher incidence of focal lesions in patients 
Table 1 General information and hormonal status of 40 patients with thyroid hemiagenesis in comparison to a control group of 80 subjects with bilobate thyroid gland.

\begin{tabular}{|c|c|c|c|}
\hline & $\begin{array}{l}\text { Thyroid } \\
\text { hemiagenesis }\end{array}$ & $\begin{array}{l}\text { Control } \\
\text { group }\end{array}$ & $P$ value \\
\hline \multicolumn{4}{|c|}{ Age of diagnosis (years) } \\
\hline Mean \pm S.D. & $37.4 \pm 17.1$ & $36.2 \pm 8.3$ & NS \\
\hline Median & 37.5 & & \\
\hline Range & $12-79$ & $12-68$ & \\
\hline \multicolumn{4}{|c|}{ Age of evaluation (years) } \\
\hline Mean \pm S.D. & $39.3 \pm 17.4$ & $36.2 \pm 8.3$ & NS \\
\hline Median & 38 & & \\
\hline Range & $13-79$ & $12-68$ & \\
\hline Gender & $35 \mathrm{~F} / 5 \mathrm{M}$ & $70 F / 10 \mathrm{M}$ & NS \\
\hline \multicolumn{4}{|c|}{$\mathrm{TSH}^{\mathrm{a}}(\mu \mathrm{IU} / \mathrm{ml} ; n$ 0.27-4.2) } \\
\hline Mean \pm s.D. & $2.37 \pm 1.4$ & $1.23 \pm 0.7$ & 0.008 \\
\hline \multicolumn{4}{|c|}{$\mathrm{FT}_{4}{ }^{\mathrm{a}}(\mathrm{pmol} / \mathrm{l} ; n$ 11.5-21.0) } \\
\hline Mean \pm s.D. & $17.9 \pm 3.9$ & $16.3 \pm 4.2$ & NS \\
\hline \multicolumn{4}{|c|}{$\mathrm{FT}_{3}^{\mathrm{a}}(\mathrm{pmol} / / ; ; n$ 3.95-6.8) } \\
\hline Mean \pm S.D. & $6.26 \pm 0.8$ & $4.88 \pm 2.1$ & 0.001 \\
\hline \multicolumn{4}{|l|}{$\mathrm{FT}_{3} / \mathrm{FT}_{4}$} \\
\hline Mean \pm s.D. & $0.35 \pm 0.1$ & $0.28 \pm 0.1$ & 0.04 \\
\hline Euthyroid & $26 / 40$ & $75 / 80$ & $<0.005$ \\
\hline Hyperthyroid & $4 / 40$ & $3 / 80$ & NS \\
\hline Hypothyroid & $10 / 40$ & $4 / 80$ & $<0.005$ \\
\hline
\end{tabular}

F, female; M, male; $n$, normal range; NS, not significant. $P>0.05$. ${ }^{\mathrm{a} C}$ Compared for patients with negative thyroid autoantibodies.

with THA was also noted. The lesions were often multiple, mixed, or solid, and were also larger than $11 \mathrm{~mm}$. Heterogeneous decreased echogenicity was more frequently observed among patients with THA as well (Table 3). Immunological assessment revealed a significantly increased prevalence of elevated thyroid autoantibodies (TRAb, TPOAb, and TgAb) in comparison to the control group (Table 4).

\section{Discussion}

Recent population-based studies estimated the prevalence of THA for $0.2-0.025 \%(2,6,15,16)$. Agenesis, for unknown reasons, concerns the left thyroid lobe in the majority of cases, $68-80 \%$ according to different reports. This is in accordance with our observations, where $87.5 \%$ of patients presented left-sided agenesis. In approximately half of the cases of THA $(70 \%$ in our series), the isthmus is present, with a characteristic hockey stick scintigraphic appearance (17). In the studied group, as well as in previous literature case reports, right lobe agenesis was in each case associated with isthmus absence. On the other hand, in most cases of left lobe agenesis, detailed sonographic imaging revealed the presence of isthmus, at least in a residual form. In conclusion, lobulation disorders in most cases result in an isolated left lobe agenesis, or associated right lobe and isthmus developmental failure.

Literature search for reports on THA reveals nearly 300 patients described so far, mostly as case studies. However, large cohorts of subjects with this anomaly have rarely been analyzed. The first review of a greater number of patients with THA, comprising 13 cases, was reported by Mariani et al. (18). However, the authors diagnosed THA entirely on the basis of scintiscans, and the patients with positive antithyroid autoantibodies were excluded in order to rule out the possibility of the absence of one thyroid lobe being a result of destructive thyroiditis. Out of the 16 patients reported by Mikosch et al., 11 had associated thyroid pathology, among which diffuse or nodular goiter was most common. A relatively high percentage ( 7 out of 16 patients) of hypothyroidism was observed due to iodine deficiency in the region of Austria, where the study was performed (3). Selection bias might be the reason for the low percentage of hypothyroidism and autoimmune thyroiditis in the research by McHenry et al. which was performed in the iodine-sufficient North American area, where a group of seven primarily surgical

Table 2 Comparison of hormonal, morphological, and immunological profiles of younger ( $\leq 25$ years) and older ( $>25$ years) patients with thyroid hemiagenesis.

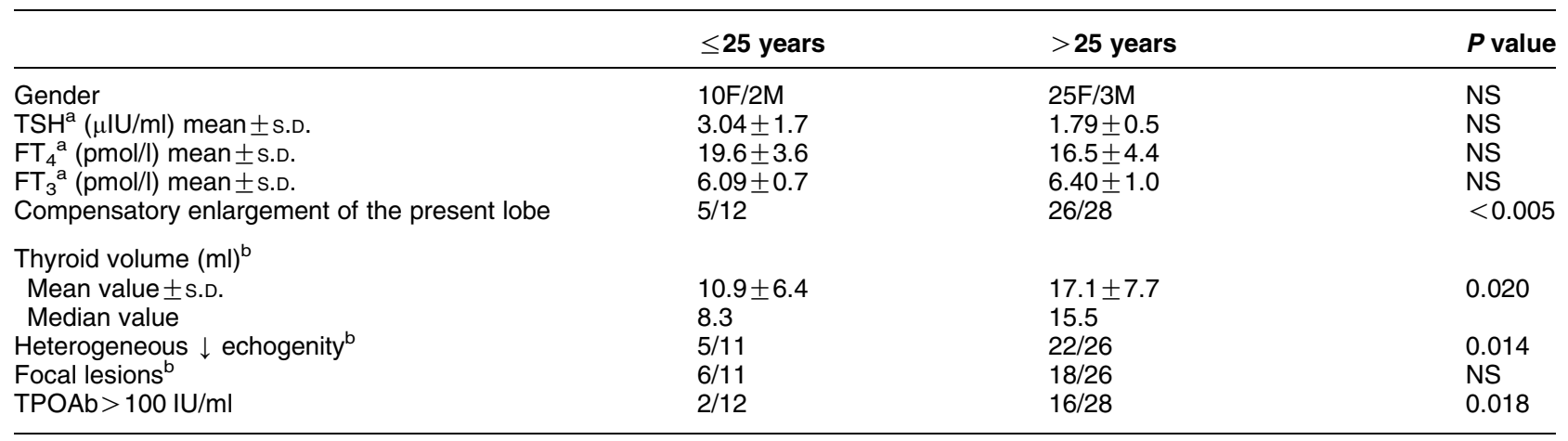

F, female; M, male; $\downarrow$, decreased; TPOAb, antithyroid peroxidase autoantibodies; $n$, normal range; NS, not significant. $P>0.05$.

${ }^{a}$ Compared for patients with negative thyroid autoantibodies.

${ }^{\mathrm{b}}$ Assessed for 37 patients in whom thyroid ultrasound was performed. 
Table 3 Ultrasound findings in 37 patients with thyroid hemiagenesis in comparison to a control group of 80 subjects with bilobate thyroid gland.

\begin{tabular}{|c|c|c|c|}
\hline & $\begin{array}{l}\text { Thyroid } \\
\text { hemiagenesis }\end{array}$ & $\begin{array}{l}\text { Control } \\
\text { group }\end{array}$ & $P$ value \\
\hline \multicolumn{4}{|l|}{ Thyroid volume (ml) } \\
\hline $\begin{array}{l}\text { Mean value } \pm \text { S.D. } \\
\text { Median value }\end{array}$ & $\begin{array}{l}15.3 \pm 7.8 \\
12.7\end{array}$ & $\begin{array}{l}15.4 \pm 8.3 \\
13.9\end{array}$ & NS \\
\hline \multicolumn{4}{|c|}{ One thyroid lobe volume (ml) } \\
\hline $\begin{array}{l}\text { Mean value } \pm \text { S.D. } \\
\text { Median value }\end{array}$ & $\begin{array}{l}15.3 \pm 7.8 \\
12.7\end{array}$ & $\begin{array}{l}7.7 \pm 4.1 \\
6.9\end{array}$ & $<0.005$ \\
\hline Focal lesions & $24 / 37$ & $23 / 80$ & $<0.005$ \\
\hline Multiple focal lesions & $15 / 37$ & $11 / 80$ & $<0.005$ \\
\hline Single focal lesion & $9 / 37$ & $12 / 80$ & NS \\
\hline Mixed/solid lesions & $23 / 37$ & $17 / 80$ & $<0.005$ \\
\hline Cystic lesions & $4 / 37$ & $9 / 80$ & NS \\
\hline $\begin{array}{l}\text { The largest lesion } \\
\leq 10 \mathrm{~mm}\end{array}$ & $10 / 37$ & $18 / 80$ & NS \\
\hline $\begin{array}{l}\text { The largest lesion } \\
>11 \mathrm{~mm}\end{array}$ & $14 / 37$ & $5 / 80$ & $<0.005$ \\
\hline $\begin{array}{l}\text { Heterogeneous } \\
\downarrow \text { echogenity }\end{array}$ & $27 / 37$ & $10 / 80$ & $<0.005$ \\
\hline
\end{tabular}

$\downarrow$, decreased; NS, not significant. $P>0.05$.

patients is described. Thus, all the patients were either euthyroid or hyperthyroid, and the associated thyroid pathological conditions were Graves' disease in two cases; colloid nodule in two cases; and simple goiter, follicular carcinoma, or follicular adenoma in the remaining three cases (1). In the study by Castanet et al., the largest series so far of 22 cases with THA was reported; however, patients with marked hypoplasia of one of the thyroid lobes were included as well. The only concomitant thyroid abnormalities described in this largely pediatric cohort were nodules and cysts, while immunological tests were not performed (19). The latest study of 14 subjects by Gursoy et al. revealed the presence of associated thyroid pathology in nine patients, of whom four patients were diagnosed with Hashimoto's thyroiditis, four patients with a nontoxic multinodular goiter, and one patient with toxic adenoma (16). The observed variety of thyroid pathological entities overlapping THA suggests a strong impact of environmental factors, age structure, and the selection of the analyzed group on the clinical profile of patients with THA.

Table 4 Thyroid autoantibodies concentrations in 40 patients with thyroid hemiagenesis in comparison to a control group of 80 subjects with bilobate thyroid gland.

\begin{tabular}{lccr}
\hline & $\begin{array}{c}\text { Thyroid } \\
\text { hemiagenesis }\end{array}$ & $\begin{array}{c}\text { Control } \\
\text { group }\end{array}$ & $\boldsymbol{P}$ value \\
\hline $\mathrm{TgAb}>60 \mathrm{IU} / \mathrm{ml}(n<60)$ & $11 / 40$ & $5 / 80$ & $<0.005$ \\
$\mathrm{TRAb}>1.5 \mathrm{IU} / \mathrm{l}(n<1.5)$ & $8 / 40$ & $4 / 80$ & 0.019 \\
$\mathrm{TPOAb}>60 \mathrm{IU} / \mathrm{ml}(n<60)$ & $20 / 40$ & $12 / 80$ & $<0.005$ \\
\hline
\end{tabular}

$\operatorname{TgAb}$, antithyroglobulin autoantibodies; TRAb, thyrotropin receptor autoantibodies; TPOAb, antithyroid peroxidase autoantibodies; $n$, normal range, NS, not significant. $P>0.05$.
The limitation of most research concerning THA is that the analyzed groups consisted of patients with associated thyroid diseases, whose thorough diagnostics led to the incidental detection of concomitant hemiagenesis. Thus, the approximated frequency might not reflect the real incidence of thyroid disorders in a healthy population. For that reason also, the preponderance of women with THA may only be a result of better detectability due to the fact that thyroid disorders occur more often in the female sex. In our series, a considerable part of patients (17 out of 40) had THA diagnosed incidentally. Most of the remaining were diagnosed due to thyroid asymmetry, which was detected by the patient or during physical examination. Fourteen of them additionally presented features of mild thyroid dysfunction on admission. However, overall prevalence of associated disorders in patients with THA was independent of the reason for thyroid ultrasound examination, and presented similarly in these subgroups.

Furthermore, our study shows that the frequency of thyroid abnormalities in patients with THA varies with age, which is probably due to the longer exposure of the hemiagenetic gland to TSH overstimulation in older patients. Along with age, individuals with a unilateral thyroid agenesis frequently develop compensatory hypertrophy of the contralateral thyroid tissue, thyroid nodular disease, and thyroid autoimmune disorders, which are usually not observed among children. This could explain the controversial conclusions about the benign character of this anomaly derived from the recent population-based studies in children $(2,6,15)$. According to these studies, no abnormalities except a significantly increased level of TSH and $\mathrm{FT}_{3}$, combined with the presence of compensatory hypertrophy described in the first paper, were found in children with THA. Additionally, our results suggest an agedependent increase in thyroid volume, which takes place along with associated decreases in TSH level.

It is still a matter of debate whether THA itself should be considered clinically insignificant, or the absence of one lobe predisposes to the development of associated thyroid disorders. The fact that diagnosis of THA is often made when some other thyroid disease is present, coupled with the paucity of cases without overt thyroid disorder under observation, makes assessment of the outcome of patients affected with this anomaly very difficult. However, the latest analysis of Gursoy et al. revealed that the incidence of THA among patients with thyroid disorders is tenfold greater than in the healthy population, supporting our conclusion that subjects with THA are more prone to develop thyroid disorders (16).

Patients with isolated THA are usually clinically euthyroid. THA results in congenital hypothyroidism only sporadically, which is detected by TSH neonatal screening (20). On the other hand, it has been observed that people with THA have significantly higher TSH and $\mathrm{FT}_{3}$ concentrations when compared to those with fully 
developed thyroid, which is in accordance with our observations and suggests an impaired functional reserve of the unilobate thyroid (6). An increased level of $\mathrm{FT}_{3}$ in comparison to subjects with bilobate gland may be explained with enhanced peripheral $\mathrm{T}_{4}-\mathrm{T}_{3}$ conversion, or stimulated thyroidal $\mathrm{T}_{3}$ secretion due to elevated TSH in response to thyroid hormones insufficiency. Nevertheless, no significant difference in $\mathrm{FT}_{4}$ concentration has been noted between the THA subjects and the control group. This indicates an altered set point of hypothalamic-pituitary-thyroid axis in patients with THA as the underdeveloped gland appears to be exposed to higher TSH overstimulation in order to preserve thyroid hormones level within normal ranges.

The recently discussed need to redefine the upper TSH limit has repercussions on the decisions concerning the treatment of patients with THA. The old reference ranges are thought to have been based on crosssectional studies of populations, uncorrected for an underlying occult thyroid disease, or any other cause for TSH elevation, while more than $95 \%$ of the population has TSH level below $2.5 \mu \mathrm{IU} / \mathrm{ml}$ (21). The true TSH normal values for formerly iodine-deficient regions were estimated at $0.25-2.12 \mu \mathrm{IU} / \mathrm{ml}$ and are distinct from the reference ranges established in areas with iodine sufficiency (22). The mean TSH level in the Caucasian population is $1.4 \mu \mathrm{IU} / \mathrm{ml}$ or even $1.18 \mu \mathrm{IU} / \mathrm{ml}$ in Afro-Americans, in whom a very low incidence of Hashimoto's thyroiditis is observed, which strongly suggests that this value is true for a healthy population (23). Baseline TSH level above $1.53 \mu \mathrm{IU} / \mathrm{ml}$ has also been reported as a predictor of subsequent thyroid dysfunction (24). Accordingly, most subjects with THA ought to be regarded as affected with subclinical hypothyroidism and patients at a higher than populational risk of developing overt thyroid failure.

Both clinical observations and experimental studies provide evidence that elevated TSH, as a thyroid growth-promoting factor, may lead to diffuse or nodular goiter and is connected with an increased risk of neoplastic transformation (25). It has been recently reported that TSH concentration at presentation is an independent predictor of the presence of thyroid malignancy, demonstrating that the risk of cancer in a thyroid nodule increases significantly with serum TSH level, even within the normal range $(26,27)$. Therefore, subjects with THA due to both high prevalence of thyroid nodular disease and an increased baseline TSH concentration are at a higher risk of developing thyroid neoplasms. Although we have diagnosed no thyroid cancer in a hemiagenetic thyroid, a high prevalence of morphological abnormalities found on US in patients with THA was demonstrated. Nodules found in these patients were predominantly solid, large $(>11 \mathrm{~mm})$, and often multiple, just opposite to the control group.

Additionally, situations of increased thyroid hormone demand (for example, during the growth and maturation period, or pregnancy, as well as environmental factors, such as iodine deficiency, or exposure to goitrogens) may further intensify TSH secretion, with all its negative consequences, and disturb the existing state of compensation in subjects with THA. This might be prevented with $\mathrm{L}-\mathrm{T}_{4}\left(\mathrm{LT}_{4}\right)$ treatment. Moreover, in cases when a thyroid of initially reduced volume is unable to satisfy the increased requirements, such treatment could avert the severe and irreversible consequences of even a slight thyroid hormone deficiency during the periods of life crucial for proper psychomotor development, such as growth, puberty, or pregnancy. Therefore, in our opinion, in patients with THA in whom treatment with $\mathrm{LT}_{4}$ was administered, a target TSH range for patients on hypothyroidism therapy $-1.0-1.5 \mu \mathrm{IU} / \mathrm{ml}$ - should also be applied.

The high prevalence of thyroid autoimmune disorders in our series is noteworthy, especially as such an immunological profile of subjects with THA has rarely been described to date and autoimmune thyroid disease is considered to manifest on the background of a genetic disposition (28). It is uncertain whether sustained overstimulation of the hemiagenetic gland in patients with THA might promote an autoimmune reaction targeted at thyroid autoantigens, and as a result, increase the risk of autoimmune thyroid disease development in genetically susceptible individuals $(29,30)$. Before 1997 , when a mandatory model of iodine prophylaxis, based on household salt iodization, was implemented, the region of Poland was classified as a moderate iodine-deficient area (31). Several authors reported that introduction of iodine supplementation was followed by an increase in the incidence of autoimmune thyroiditis (32-34). Nevertheless, significantly higher prevalence of thyroid autoimmune disorders in comparison to a control group of subjects with bilobate thyroid, living in the same area and matched for age and gender, requires further investigation.

In conclusion, our study provides the first systematic analysis of a large group of subjects with THA. Although hormonal production of a single thyroid lobe was usually found to be sufficient to maintain clinical euthyroidism, significantly higher than populational TSH and $\mathrm{FT}_{3}$ levels are observed among the subjects. Furthermore, a higher incidence of associated thyroid pathologies, including thyroid autoimmune disorders, disturbances in thyroid function, and thyroid morphological abnormalities was noted among patients with THA when compared to subjects with fully developed thyroid gland. Additionally, the frequency of some pathologies in subjects with THA increased significantly with age. The obtained results suggest that individuals diagnosed with THA are more likely to develop thyroid pathology, while sustained TSH overstimulation of the undersized gland might play a major role in its etiopathogenesis. Therefore, diagnosis of THA, in our opinion, should be followed by systematic 
monitoring of thyroid morphology and hormonal function. Furthermore, as a preventive measure or treatment, adequate $\mathrm{LT}_{4}$ substitution ought to be considered in patients with an elevated TSH level. However, the outcomes of such an intervention should be investigated in the future before a firm recommendation can be made.

\section{Declaration of interest}

There is no conflict of interest that could be perceived as prejudicing the impartiality of the research reported.

\section{Funding}

This study was supported by the State Committee for Scientific Research (grant Nr N N402 016736).

\section{Acknowledgements}

W Szaflarski acknowledges support from the START Fellowship of the Foundation for Polish Science.

\section{References}

1 McHenry CR, Walfish PG, Rosen IB, Lawrence AM \& Paloyan E. Congenital thyroid hemiagenesis. American Surgeon 199561 634-638 (discussion 638-639).

2 Shabana W, Delange F, Freson M, Osteaux M \& De Schepper J. Prevalence of thyroid hemiagenesis: ultrasound screening in normal children. European Journal of Pediatrics $2000 \mathbf{1 5 9}$ 456-458.

3 Mikosch P, Gallowitsch HJ, Kresnik E, Molnar M, Gomez I \& Lind P. Thyroid hemiagenesis in an endemic goiter area diagnosed by ultrasonography: report of sixteen patients. Thyroid 19999 1075-1084.

4 Pizzini AM, Papi G, Corrado S, Carani C \& Roti E. Thyroid hemiagenesis and incidentally discovered papillary thyroid cancer: case report and review of the literature. Journal of Endocrinological Investigation 200528 66-71.

5 Park IH, Kwon SY, Jung KY \& Woo JS. Thyroid hemiagenesis: clinical significance in the patient with thyroid nodule. Journal of Laryngology and Otology 2006120 605-607.

6 Maiorana R, Carta A, Floriddia G, Leonardi D, Buscema M, Sava L, Calaciura F \& Vigneri R. Thyroid hemiagenesis: prevalence in normal children and effect on thyroid function. Journal of Clinical Endocrinology and Metabolism 200388 1534-1536.

7 Deladoey J, Vassart G \& Van Vliet G. Possible non-Mendelian mechanisms of thyroid dysgenesis. Endocrine Development 200710 29-42.

8 Tsang SK \& Maher J. Thyroid hemiagenesis accompanying a thyroglossal duct cyst: a case report. Clinical Nuclear Medicine 1998 23 229-232.

9 Sharma R, Mondal A, Popli M, Sahoo M, Malhotra N \& Soni S. Hemiagenesis of the thyroid associated with chronic lymphocytic thyroiditis. Clinical Nuclear Medicine 200126 506-508.

10 Nakamura S, Isaji $M$ \& Ishimori $M$. Thyroid hemiagenesis with postpartum silent thyroiditis. Internal Medicine $2004 \mathbf{4 3}$ 306-309.

11 Ozgen AG, Saygili F \& Kabalak T. Thyroid hemiagenesis associated with Graves' disease and Graves' ophthalmopathy: case report. Thyroid $20041475-77$.
12 Lee YS, Yun JS, Jeong JJ, Nam KH, Chung WY \& Park CS. Thyroid hemiagenesis associated with thyroid adenomatous hyperplasia and papillary thyroid carcinoma. Thyroid $2008 \mathbf{1 8}$ 381-382.

13 Ruchala M, Szczepanek E, Skiba A, Czepczynski R \& Sowinski J. Graves' hyperthyroidism following primary hypothyroidism due to Hashimoto's thyroiditis in a case of thyroid hemiagenesis: case report. Neuroendocrinology Letters 200829 55-58.

14 Andersson M, Takkouche B, Egli I, Allen HE \& de Benoist B. Current global iodine status and progress over the last decade towards the elimination of iodine deficiency. Bulletin of the World Health Organization 200583 518-525.

15 Korpal-Szczyrska M, Kosiak W \& Swieton D. Prevalence of thyroid hemiagenesis in an asymptomatic school children population. Thyroid 200818 637-639.

16 Gursoy A, Anil C, Unal AD, Demirer AN, Tutuncu NB \& Erdogan MF. Clinical and epidemiological characteristics of thyroid hemiagenesis: ultrasound screening in patients with thyroid disease and normal population. Endocrine 200833 338-341.

17 Melnick JC \& Stemkowski PE. Thyroid hemiagenesis (hockey stick sign): a review of the world literature and a report of four cases. Journal of Clinical Endocrinology and Metabolism $1981 \mathbf{5 2}$ 247-251.

18 Mariani G, Molea N, Toni MG \& Bianchi R. Thyroid hemiagenesis: a review of thirteen consecutive cases. Journal of Nuclear Medicine and Allied Sciences 198024 183-187.

19 Castanet M, Leenhardt L, Leger J, Simon-Carre A, Lyonnet S, Pelet A, Czernichow P \& Polak M. Thyroid hemiagenesis is a rare variant of thyroid dysgenesis with a familial component but without Pax8 mutations in a cohort of 22 cases. Pediatric Research $2005 \mathbf{5 7} 908-913$.

20 Ramos HE, Nesi-Franca S, Boldarine VT, Pereira RM, Chiamolera MI, Camacho CP, Graf H, de Lacerda L, Carvalho GA \& Maciel RM. Clinical and molecular analysis of thyroid hypoplasia: a population-based approach in Southern Brazil. Thyroid 200919 61-68.

21 Baloch Z, Carayon P, Conte-Devolx B, Demers LM, Feldt-Rasmussen U, Henry JF, LiVosli VA, Niccoli-Sire P, John R, Ruf J, Smyth PP, Spencer CA \& Stockigt JR. Laboratory medicine practice guidelines. Laboratory support for the diagnosis and monitoring of thyroid disease. Thyroid 200313 3-126.

22 Volzke H, Alte D, Kohlmann T, Ludemann J, Nauck M, John U \& Meng W. Reference intervals of serum thyroid function tests in a previously iodine-deficient area. Thyroid $2005 \mathbf{1 5}$ 279-285.

23 Hollowell JG, Staehling NW, Flanders WD, Hannon WH, Gunter EW, Spencer CA \& Braverman LE. Serum TSH, T(4), and thyroid antibodies in the United States population (1988 to 1994): National Health and Nutrition Examination Survey (NHANES III). Journal of Clinical Endocrinology and Metabolism 200287 489-499.

24 Warren RE, Perros P, Nyirenda MJ \& Frier BM. Serum thyrotropin is a better predictor of future thyroid dysfunction than thyroid autoantibody status in biochemically euthyroid patients with diabetes: implications for screening. Thyroid $2004 \mathbf{1 4}$ 853-857.

25 Pedrinola F, Rubio I, Santos CL \& Medeiros-Neto G. Overexpression of epidermal growth factor and epidermal growth factorreceptor mRNAs in dyshormonogenetic goiters. Thyroid 2001 11 15-20.

26 Boelaert K, Horacek J, Holder RL, Watkinson JC, Sheppard MC \& Franklyn JA. Serum thyrotropin concentration as a novel predictor of malignancy in thyroid nodules investigated by fine-needle aspiration. Journal of Clinical Endocrinology and Metabolism 2006 91 4295-4301.

27 Haymart MR, Repplinger DJ, Leverson GE, Elson DF, Sippel RS, Jaume JC \& Chen H. Higher serum thyroid stimulating hormone level in thyroid nodule patients is associated with 
greater risks of differentiated thyroid cancer and advanced tumor stage. Journal of Clinical Endocrinology and Metabolism 200893 809-814.

28 Weetman AP. The genetics of autoimmune thyroid disease. Hormone and Metabolic Research 200941 421-425.

29 Rapoport B \& McLachlan SM. Thyroid autoimmunity. Journal of Clinical Investigation $2001 \mathbf{1 0 8} 1253-1259$.

30 Flynn JC, Gilbert JA, Meroueh C, Snower DP, David CS, Kong YC \& Banga JP. Chronic exposure in vivo to thyrotropin receptor stimulating monoclonal antibodies sustains high thyroxine levels and thyroid hyperplasia in thyroid autoimmunity-prone HLADRB1*0301 transgenic mice. Immunology 2007122 261-267.

31 Szybinski Z, Delange F, Lewinski A, Podoba J, Rybakowa M, Wasik R, Szewczyk L, Huszno B, Golkowski F, Przybylik-Mazurek E, Karbownik M, Zak T, Pantoflinski J, Trofimiuk M \& Kinalska I. A programme of iodine supplementation using only iodised household salt is efficient - the case of Poland. European Journal of Endocrinology 2001144 331-337.
32 Baczyk M, Ruchala M, Pisarek M, Pietz L, Wrotkowska E, Wojewoda-Korbelak M, Dziubandowska A, Gembicki M \& Sowinski J. Iodine prophylaxis in children population on the Wielkopolska Region area from year 1992 to 2005 . Endokrynologia Polska 200657 110-115.

33 Teng W, Shan Z, Teng X, Guan H, Li Y, Teng D, Jin Y, Yu X, Fan C, Chong W, Yang F, Dai H, Yu Y, Li J, Chen Y, Zhao D, Shi X, Hu F, Mao J, Gu X, Yang R, Tong Y, Wang W, Gao T \& Li C. Effect of iodine intake on thyroid diseases in China. New England Journal of Medicine $20063542783-2793$.

34 Fountoulakis S, Philippou G \& Tsatsoulis A. The role of iodine in the evolution of thyroid disease in Greece: from endemic goiter to thyroid autoimmunity. Hormones 20076 25-35.

Received 1 October 2009

Accepted 21 October 2009 